\title{
Relato de Concepção de Aplicação para Gestão da Saúde da Pessoa Idosa Dependente
}

\author{
Ivana Cristina de Holanda Cunha Barreto ${ }^{1}$, Luiz Odorico Monteiro de Andrade, \\ Aline Luiza de Paulo Evangelista ${ }^{1}$, Ismayle de Sousa Santos ${ }^{2}$, Rossana Maria de \\ Castro Andrade ${ }^{2}$, Antonio Mauro Barbosa de Oliveira ${ }^{3}$, João Bastos Freire Neto ${ }^{1}$, \\ Virna Maria Aguiar Frota ${ }^{1}$
}

${ }^{1}$ Fundação Oswaldo Cruz (Fiocruz) - Rua São José, s/n, Precabura, CEP 61.760-000 Eusébio - CE - Brasil

${ }^{2}$ Universidade Federal do Ceará (UFC) - Fortaleza - CE Brasil

${ }^{3}$ Instituto Federal de Educação, Ciência e Tecnologia do Ceará (IFCE) - Aracati, CE Brasil

\begin{abstract}
\{ivana_barreto@yahoo.com.br,odorico0811@gmail.com, asalineluiza@gmail.com, ismaylesantos@great.ufc.br, rossana@ufc.br, amauroboliveira@gmail.com,joaobastosgeriatra@gmail.com, virna.a.frota@gmail.com\}
\end{abstract}

Abstract. Despite the increase in the number of elderly people and health-care applications, there is still a lack of applications aimed at managing the care of elderly people. In this context, this paper aims to report the development of an application for the use of the Health Professional dedicated to the management of the health of dependent elderly people. This paper discusses the formation of an interdisciplinary team of health and computing professionals. As a process model for the development of the application, the Scrum was chosen and ten functionalities were elicited, among them: Clinical and Functional Evaluation; Care Plan; Medicine Administration; Visit Registration; Intercurrences; Notifications and Alerts.

Resumo. Apesar do aumento do número de pessoas idosas e das aplicações voltadas para saúde, ainda existe uma carência por aplicações voltadas a gestão do cuidado de pessoas idosas. Neste contexto, o objetivo deste artigo é descrever o desenvolvimento de uma aplicação para uso do Profissional de Saúde dedicada à gestão da saúde da pessoa idosa dependente. Discute-se no artigo a formação de uma equipe interdisciplinar de profissionais de saúde e de computação. Como modelo de processo para o desenvolvimento da aplicação, optou-se pelo método ágil Scrum e dez funcionalidades foram elicitadas, dentre elas: Avaliação Clínica e Funcional; Plano de Cuidados; Medicamentos, Registro de Visitas; Intercorrências, Notificações e Alertas.

\section{Introdução e Justificativa}

A evolução científica e tecnológica possibilitou novos conhecimentos sobre envelhecimento humano, diagnósticos e tratamentos que propiciaram crescimento da expectativa de vida das pessoas em todo o mundo. A expectativa de vida passou de 62,7 anos em 1980 para 75,8 anos em 2019 e poderá chegar a 81,3 anos em 2050, gerando aumento significativo na população de pessoas idosas [Brasil, 2008], o que repercutirá no aumento de custos para os 
sistemas de saúde. De acordo com a Pesquisa Nacional de Saúde de 2013, 30,1\% das pessoas idosas apresentam pelo menos uma limitação para as atividades da vida diária [Brasil, 2015], os desafios para o cuidado dessa população são significativos, necessitando de recursos humanos qualificados.

Em janeiro de 2020, a Organização Mundial da Saúde (OMS) publicou o documento Estratégia Global para Saúde Digital 2020-2024 conceituando Saúde Digital como o campo de conhecimento e prática associado ao desenvolvimento e ao uso das tecnologias digitais para melhorar a saúde [WHO, 2020]. A Saúde Digital expande o conceito de eSaúde para incluir os consumidores digitais com uma maior variedade de dispositivos inteligentes e equipamentos conectados. Também abrange outros usos das tecnologias digitais para a saúde, como Internet das Coisas (IoT), Inteligência Artificial, Big Data Analytics e robótica, tendo o potencial de melhorar a saúde significativamente por meio do aumento da eficiência do diagnóstico médico, de tratamentos baseados em evidências e do suporte ao autocuidado, fortalecendo a atenção à saúde.

O uso de ferramentas tecnológicas na saúde da pessoa idosa aqui pautado referencia-se pelo conceito de saúde digital da OMS junto à classificação das intervenções em Saúde Digital [WHO, 2018], confirmando as diferentes maneiras pelas quais as tecnologias digitais podem auxiliar os sistemas de saúde, objetivando promover uma linguagem acessível e vinculativa para o planejamento dos sistemas de saúde.

Diante do panorama do envelhecimento no Brasil [Brasil, 2008], e, particularmente, da elevada prevalência de dependência para as atividades da vida diária na população idosa, o projeto em andamento de pesquisa e desenvolvimento tecnológico propõe uma estratégia de cuidado dessa população por meio de uma ferramenta de Saúde Digital com o objetivo de apoiar tanto profissionais de saúde quanto cuidadores/familiares envolvidos na atenção à saúde da pessoa idosa, considerando a interdisciplinaridade presente na interação entre profissionais de saúde e profissionais da engenharia de software para a qualidade do produto que está sendo desenvolvido.

Desse modo, este trabalho tem por objetivo descrever a concepção de uma aplicação para uso do Profissional de Saúde dedicada à gestão da saúde da pessoa idosa dependente e seus resultados preliminares.

\section{Metodologia}

O desenvolvimento da aplicação segue a metodologia Scrum [Schwaber e Sutherland, 2013] com entregas baseadas em Sprints e story points. Durante o desenvolvimento estão sendo realizadas as reuniões previstas no Scrum: (i) Reunião Diária; (ii) Reunião de Revisão; e (iii) Reunião de Retrospectiva, as três reuniões com foco nas entregas incrementais do produto e em sua qualidade [Sutherland e Sutherland, 2018].

Além disso, o desenvolvimento de uma tecnologia aplicada à saúde requer uma equipe interdisciplinar [Sommerville, 2015]. No caso da tecnologia aqui apresentada, foram estruturados dois times. O time da tecnologia com User eXperience - UX, User Interface UI, Requisitos, Desenvolvimento e Testes. O time da saúde com stakeholders, incluindo dois médicos sanitaristas, um geriatra, uma terapeuta ocupacional e uma enfermeira sanitarista. Foi também designada uma Product Owner (P.O.) e um Scrum Master, funções essenciais no Scrum. 
No intuito de refinar os requisitos do módulo Profissional de Saúde, bem como garantir uma experiência do usuário adequada, estudos foram realizados pelos integrantes do projeto de pesquisa para nortear o processo de desenvolvimento da solução tecnológica aqui relatado [Almeida et al., 2019; Araújo et al.; 2020], evidenciando as características essenciais, ou seja, que agregam valor ao produto, incluindo os requisitos necessários e as necessidades dos profissionais de saúde no cuidado da pessoa idosa.

Esse conjunto de pesquisas auxiliou na especificação e validação dos requisitos para que fosse iniciada a etapa de desenvolvimento. A partir das pesquisas realizadas e da metodologia ágil aplicada, são apresentados alguns resultados preliminares, mais especificamente, sobre o Módulo Profissional de Saúde, primeiro em andamento, tendo em vista que o mesmo será integrado ao Módulo Cuidador/Familiar, consistindo na Plataforma Zelo Saúde.

\section{Resultados preliminares e discussão}

Considerando o método ágil Scrum, durante o ano de 2019, concretizaram-se 15 sprints, iniciando a Sprint 0 em maio de 2019 e concluindo a Sprint 14 em dezembro do mesmo ano. As sprints contaram com duração de 2 ou 3 semanas, ficando a critério dos times envolvidos a definição, a considerarem aspectos decisivos, tais como: complexidade de casos de uso a serem desenvolvidos, feriados e imprevistos de força maior.

Para o desenvolvimento da aplicação foi preciso refinar requisitos, elaborar mockups, realizar sprints e testes. As atividades foram iniciadas tão logo fossem validados os mockups por P.O. e stakeholders. Com a validação destes mockups, os casos de uso puderam ser refinados e encaminhados para os desenvolvedores. No total, foram 17 requisitos, 89 casos de uso especificados e 28 regras de negócio (restrições ou premissas). Esses requisitos, casos de uso e regras de negócio foram documentados na ferramenta de gerenciamento de projetos Redmine ${ }^{1}$.

O Módulo Profissional de Saúde, que se encontra em fase final de desenvolvimento, utiliza o framework React Native ${ }^{2}$, escolhido por facilitar o desenvolvimento híbrido utilizando um mesmo código fonte. Todas as funcionalidades a nível de aplicativo para os usuários finais estão sendo desenvolvidas utilizando esse framework. Durante o processo de desenvolvimento existe uma preocupação constante com questões de reusabilidade de código [Younoussi e Roudies, 2015] e com a manutenibilidade da aplicação [Martin, 2009], além de outras questões, como desempenho. O servidor está sendo desenvolvido em NodeJS ${ }^{3}$, framework poderoso e flexível que fornece diversos recursos para construção de APIs (Application Programming Interface) robustas de acesso a banco de dados.

O time de desenvolvimento visualizou que para o contexto do projeto era necessário tornar o servidor uma API multiserviços, assim, foi planejado e construído a arquitetura necessária para tal, ficando uma API serviço para banco de dados relacional e outra para o banco de dados não relacional, este último responsável por armazenar os dados de históricos gerados pelo referido módulo. O time também configura e mantém um versão estável do

\footnotetext{
1 https://www.redmine.org/

2 http://www.reactnative.com/

3 https://nodejs.org/en/
} 
servidor na nuvem (Amazon Web Service ${ }^{4}$ ) que é utilizado pelo time de testes e requisitos para os reports de testes das versões de release do aplicativo.

Durante o design de interface, foram realizados planejamento, desenvolvimento e utilização do protótipo de alta fidelidade, composto pelas seguintes funcionalidades a partir dos requisitos levantados inicialmente: cadastro/remoção/edição dos dados da pessoa idosa, cadastro/remoção/edição dos dados do profissional de saúde, cadastro/edição da Avaliação Clínica e Funcional, geração automática/edição do Plano de Cuidados, cadastro/remoção/edição da lista de medicamentos, suspeita de maus-tratos, emissão/recebimento de notificações e alertas, cadastro/visualização do registro de visitas, cadastro/visualização de intercorrências, e geração de relatório.

Quando o profissional de saúde vincula o perfil da pessoa idosa (Figura 1.a) é possível contar com Dados Pessoais, Avaliação Clínica e Funcional e Plano de Cuidados. Na Avaliação Clínica e Funcional, o profissional de saúde identifica as dependências, os problemas e as doenças de determinada pessoa idosa, gerando automaticamente o Plano de Cuidados com a relação de ações e instruções necessárias para o cuidado em saúde.

Outras funcionalidades que o profissional de saúde pode contar ao vincular o perfil da pessoa idosa são (Figura 1.b): registrar as visitas realizadas em domicílio; cadastrar os medicamentos utilizados; inserir suspeita de maus-tratos; informar intercorrências, gerando alertas sobre as mesmas, bem como outros alertas e também notificações; contactar a equipe de saúde associada à pessoa idosa e gerar relatórios.

Assim, o principal resultado alcançado durante essa jornada do ano de 2019 foi a implementação de funcionalidades que deixam o sistema apto para avaliação das expectativas dos stakeholders. O público alvo da solução tecnológica a ser alcançado é composto por profissionais de saúde e cuidadores/familiares de pessoas idosas dependentes. Parte do produto desenvolvido já agrega valor na gestão da saúde da pessoa idosa dependente devido às funcionalidades necessárias ao cotidiano de trabalho dos profissionais de saúde.

${ }^{4}$ https://aws.amazon.com/pt/ 


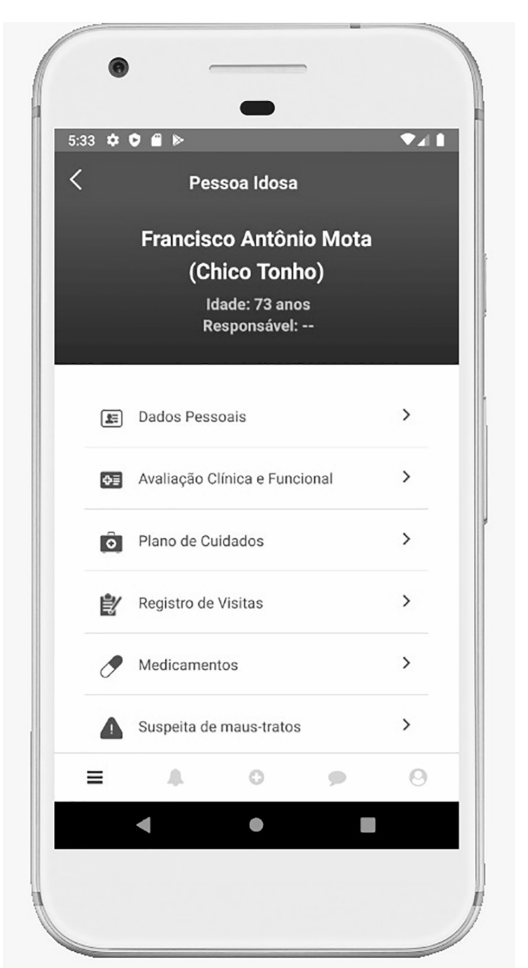

(a)

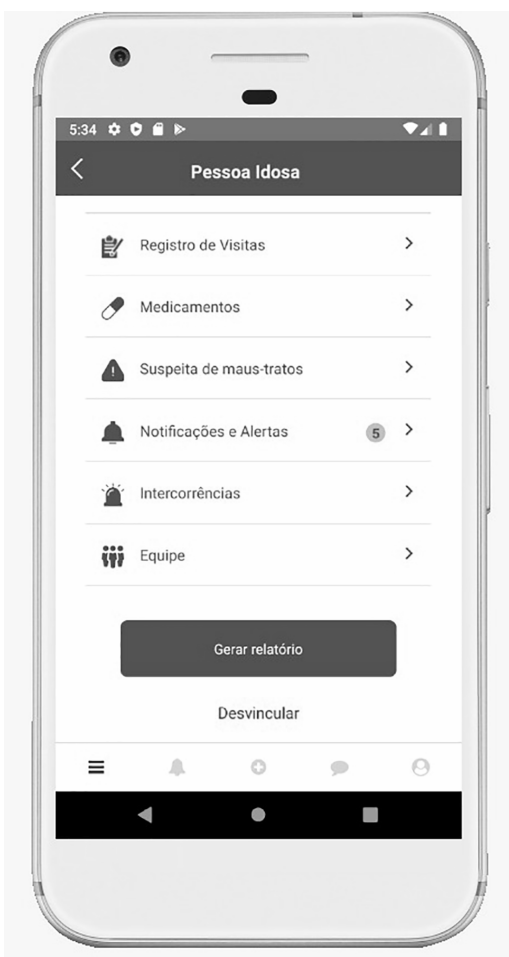

(b)

Figura 1: Telas da aplicação: Interface com funcionalidades no perfil da pessoa idosa vinculada ao profissional de saúde.

\section{Considerações Finais}

Como ações que promoveram o bom desenvolvimento do trabalho estão: realização de reuniões e workshops de alinhamento da equipe para as atividades; designação de uma P.O. para mediar as relações entre as equipes de tecnologia e saúde; realização sprints no método Scrum; organização da comunicação dos grupos por meio do Slack ${ }^{5}$.

Entre os benefícios alcançados, destacam-se: criação de fluxos padronizados; escolha de melhores opções para as funcionalidades a partir das necessidades dos stakeholders; retroalimentação das regras de negócios e resolução de impedimentos. As etapas seguintes consistem na validação de requisitos e mockups do Módulo Cuidador/Familiar, bem como a sua integração ao Módulo Profissional de Saúde para finalização da versão beta da Plataforma Zelo Saúde e início da prova de conceito a ser realizada nos municípios de Fortaleza e Eusébio, ambos no Estado do Ceará

\section{Agradecimentos}

Ao Programa Inova Fiocruz pelo suporte financeiro (projeto VPPIS-004-FIO-18); ao CNPQ pela bolsa de produtividade DT-2 de Rossana Maria de Castro Andrade ( $\mathrm{N}^{\circ}$ do processo 315543/2018-3); aos bolsistas do projeto: Bruno Teixeira de Sousa, Ítalo Linhares de Araújo, Joseane de Oliveira Vale Paiva, Paula Caldas Melo, Paulo Artur de Sousa Duarte e Rômulo Gadelha de Moura Lima.

5 https://slack.com/intl/pt-br/ 


\section{Referências}

Almeida, R. L. A. ; Paiva, J. O. V. ; Gouveia, T. ; Barroso, H. ; Bastos, J. ; Santos, I. S. ; Evangelista, A. ; Andrade, L. O. M. ; Barreto, I. C. H. C. ; Andrade, R. M. C. . (2019) "Fictitious personas in the integration of an interdisciplinary team in an older adults health application project". In: Anais do XVIII Simpósio Brasileiro de Qualidade de Software, 2019, Fortaleza, CE.

Araujo, I. L. ; Costa Junior, E. ; Duarte, P. A. S. ; Santos, I. S. ; Oliveira, P. A. M. ; mendes, C. M. O. ; Andrade, R. M. C. ; Barreto, I. C. H. C. ; Andrade, L. O. M. (2020) "Towards a Taxonomy for the Development of Older Adults Healthcare Applications". In: Proceedings of the 53rd Hawaii International Conference on System Sciences (HCISS).

Brasil. Ministério do planejamento, orçamento e gestão. Instituto Brasileiro de Geografia e Estatística (IBGE). (2008) "Projeção da população do Brasil por sexto e idade:1980-2050", http://www.nescon.medicina.ufmg.br/biblioteca/registro/Projecao_da_populacao_do_Brasil _por_sexo_e_idade_1980_2050_/568.

Brasil. Instituto Brasileiro de Geografia e Estatística (IBGE). (2015) "Pesquisa Nacional de Saúde 2013: ciclos de vida", http://biblioteca.ibge.gov.br/visualizacao/livros/liv94522.pdf.

Martin, Robert C. (2009) "Código Limpo: Habilidades Práticas do Agile Software. Rio de Janeiro: Alta Books". Tradução de Clean Code: A Handbook of Agile Software.

Schwaber, K. and Sutherland, J. (2013) “"'The Scrum Guide - The Definitive Guide to Scrum: The Rules of the Game", http://www.Scrumguides.org/docs/Scrumguide/v1/Scrum-guideus.pdf.

Sommerville, I (2015). Software Engineering. 10th edição. Addison-Wesley

Sutherland, J. and Sutherland, J.J. (2018), Scrum: a arte de fazer o dobro do trabalho na metade do tempo, tradução Nina Lua, $3^{a}$ edição.

World Health Organization (WHO). (2018)"Classification of digital health interventions v1.0. A shared language to describe the uses of digital technology for health", http://www.who.int/reproductivehealth/publications/mhealth/classification-digital-healthinterventions/en/.

World Health Organization. Global Strategy on Digital Health [Internet]. 2020. Available at: www.who.int/health-topics/digital-health\#tab=tab_1.

Younoussi, S. e Roudies, O (2015). "All about software reusability: a systematic literature review ". Journal of Theoretical and Applied Information Technology n 76, p. 64-75 